\title{
Placental lipid processing in response to a maternal high-fat diet and diabetes in rats
}

\author{
Eli J. Louwagie ${ }^{1}$, Tricia D. Larsen ${ }^{2}$, Angela L. Wachal ${ }^{2}$ and Michelle L. Baack ${ }^{2}$
}

BACKGROUND: Diabetes and obesity during pregnancy have an impact on the health of both mothers and developing babies. Prevention focuses on glycemic control, but increasing evidence implicates a role for lipids. Using a rat model, we showed that a maternal high-fat (HF) diet increased perinatal morbidity and mortality, but lipid processing across the maternal-placental-fetal triad remained unstudied. We hypothesized that HF diet would disrupt placental lipid processing to exaggerate fuel-mediated consequences of diabetic pregnancy.

METHODS: We compared circulating lipid profiles, hormones, and inflammatory markers in dams and rat offspring from normal, diabetes-exposed, HF-diet-exposed, and combinationexposed pregnancies. Placentae were examined for lipid accumulation and expression of fuel transporters.

RESULTS: Maternal HF diet exaggerated hyperlipidemia of pregnancy, with diabetes marked dyslipidemia developed in dams but not in offspring. Placentae demonstrated lipid accumulation and lower expression of fatty acid (FA) transporters. Diet-exposed offspring had a lower fraction of circulating essential FAs. Pregnancy loss was significantly higher in dietexposed but not in diabetes-exposed pregnancies, which could not be explained by differences in hormone production. Although not confirmed, inflammation may play a role.

CONCLUSION: Maternal hyperlipidemia contributes to placental lipid droplet accumulation, perinatal mortality, and aberrant FA profiles that may influence the health of the developing offspring.

D iabetes and obesity are increasingly common $(1,2)$, and during pregnancy are associated with increased circulating metabolic fuels that can have a negative impact on the health of both the mother and developing fetus $(3,4)$. Women with diabetes have higher rates of pregnancy-related complications including miscarriages and stillbirths (5-8). Their infants have higher rates of complications in the neonatal period $(7,9)$ and throughout life $(10)$. The primary preventative measure is optimizing glycemic control (11), yet women with good glycemic control and non-diabetic obese women have increased perinatal morbidity and so do their infants $(5,7,9,12)$. This implicates dyslipidemia as a mediating factor. Using a rat model, our laboratory has demonstrated that a maternal high-fat (HF) diet ( $40 \%$ vs. $18 \%$ dietary fat) exacerbates the physiologic hyperlipidemia of pregnancy and increases morbidity and mortality in newborn offspring $(13,14)$. However, the placental response to maternal dyslipidemia remains poorly understood. We hypothesized that a maternal HF diet would exaggerate fuel-mediated consequences of diabetic pregnancy by disrupting placental lipid processing, which we defined as the collective actions of lipid transport, storage, and use of fats for production of hormones or inflammatory precursors.

Lipid transport supports the increased metabolic needs of mother, placenta, and baby. As a normal pregnancy progresses, it is accompanied by a physiologic hyperlipidemia with increasing maternal-fetal transport of essential fatty acids (FAs) to support fetal growth and brain development (15). The fetus depends on a maternal source for essential long-chain polyunsaturated FA (LCPUFAs) including arachidonic acid (ARA) and docosahexaenoic acid (15). Increasing evidence suggests that infants born to diabetic mothers have lower levels of LCPUFAs (16-18) purportedly from impaired maternal-fetal transport. In addition to facilitating nutrient transport, the placenta is the principal metabolic, respiratory, excretory, and endocrine organ of pregnancy (19). The placenta must store lipids as important precursors of hormones and inflammatory mediators that support a healthy pregnancy. Therefore, the relationship between maternal dyslipidemia and impaired placental function may have extensive consequences for pregnancy and infant health.

To test our hypothesis, we used a rat model to determine whether a maternal HF diet, especially alongside late-gestation diabetes, independently or additively impedes maternalplacental-fetal lipid processing as indicated by lower LCPUFA levels in offspring, decreased placental hormone production, and/or increased markers of inflammation.

\section{METHODS}

\section{Animal Care}

This study followed the guidelines from the Animal Welfare Act and the National Institutes of Health Guide for the Care and Use of Laboratory Animals and was under approval from the Sanford

\footnotetext{
${ }^{1}$ Biology Department, Augustana University, Sioux Falls, South Dakota, USA; ${ }^{2}$ Children's Health Research Center, Sanford Research, Sioux Falls, South Dakota, USA. Correspondence: Michelle L. Baack (michelle.baack@sanfordhealth.org)

Received 17 April 2017; accepted 23 October 2017; advance online publication 3 January 2018. doi:10.1038/pr.2017.288
} 


\section{Placental response to hyperlipidemia $\quad$ Articles}

Research Institutional Animal Care and Use Committee. Model characteristics have been described previously $(13,14)$. In brief, female Sprague-Dawley rats (Harlan Laboratories, Indianapolis, IN) received control (TD2018 Teklad; Harlan Laboratories, Madison, WI) or HF (TD95217 custom diet Teklad; Harlan Laboratories) diet for at least 28 days before breeding. On embryonic day (E) 14 of a timed pregnancy, dams received $0.09 \mathrm{M}$ citrate buffer or $65 \mathrm{mg} / \mathrm{kg}$ of streptozocin (Sigma Life Sciences, St Louis, MO) in citrate buffer to induce diabetes. Thereafter, twice-daily, sliding-scale insulin was administered to keep non-fasting glucose levels in a target range of $200-400 \mathrm{mg} / \mathrm{dl}$. Diabetic criteria for rats are similar to those of humans (20); rats that did not reach a blood glucose $\geq 200 \mathrm{mg} / \mathrm{dl}$ following streptozocin administration were excluded from this study. Dams underwent C-section (under isoflurane:oxygen anesthesia) on E21 for placental collection or were allowed to deliver spontaneously to yield postnatal day $(\mathrm{P}) 1$ offspring from four distinct groups: controls, diabetes-exposed, diet-exposed, and combination-exposed.

Animals were housed in a temperature-controlled, light-dark cycled facility with free access to water and food. Euthanasia occurred under isofluorane:oxygen induction followed by cervical dislocation for newborn offspring or cardiac puncture/heart removal for older animals. Physical locations of the placentae and embryos were recorded as being from the left or right uterine horn and were numbered consecutively by proximity to the cervix. Placentae were snap-frozen and stored at $-80^{\circ} \mathrm{C}$. Perinatal losses were assessed by inspection of uterine horns, which included counting placentations alongside the number of delivered pups (dead or alive) and retained stillbirths.

\section{Venous Blood Sampling}

Maternal blood was collected at baseline, after 28 days of specified diet, E14, and delivery (E21 with C-section and P1 with spontaneous delivery). While under anesthesia for maternal c-section, whole blood was collected from jugular veins of E21 offspring before umbilical cord or placental separation and euthanasia. In P1 offspring blood was collected by jugular venipuncture immediately after being killed. Whole blood and plasma fraction aliquots were stored at $-80^{\circ} \mathrm{C}$.

\section{Triacylglycerol and Non-Esterified FA Analyses}

Plasma triacylglycerol (TG) levels were measured using a Triglyceride Colorimetric Assay Kit (ThermoFisher Scientific, Waltham, MA) via the manufacturer's instructions. A dilution curve was created using TG standards (ThermoFisher Scientific) to verify assay precision. Plasma non-esterified FA (NEFA) levels were measured using a NEFA-HR Colorimetric Kit (Wako Diagnostics, Richmond, $\mathrm{VA}$ ) via the manufacturer's instructions. Absorbance for TG and NEFA analyses was measured using SpectroMax Plus (Molecular Devices, Sunnyvale, CA).

\section{FA Analyses}

Whole-blood FA analysis was performed using gas chromatography following direct transesterification of whole blood using $14 \%$ borontrifluoride methanol and hexane solution with a quantitative internal standard (17:0 heptadecanoic acid) at $100{ }^{\circ} \mathrm{C}$ for $10 \mathrm{~min}$. After cooling, high-performance liquid chromatography-grade water was added and the sample was vortexed and centrifuged for phase separation. FA methyl-esters in hexane were analyzed alongside quantitative external standard (15:0 methylpentadecanoate) using capillary gas chromatography on an Agilent 7890A (Agilent Technologies, Santa Clara, CA) equipped with an Agilent CP7489 Capillary Column $(100 \mathrm{~m}$ length $\times 0.25 \mathrm{~mm}$ internal diameter $\times$ $0.36 \mathrm{~mm}$ outer diameter, $0.20 \mu \mathrm{m}$ film thickness) using hydrogen as carrier gas. FAs of 10-24 carbons were detected with flame ionization detector and recorded using ChemStation interface system (Agilent Technologies). Peaks were measured using Open LAB Chromatography Data System and corresponding authentic standard, 37 FAME mix (Sigma-Aldrich, St Louis, MO).

\section{Placental Lipid Analyses}

Whole placentae, weighing $0.49-1.78 \mathrm{~g}$, were snap-frozen and stored at $-80^{\circ} \mathrm{C}$. Lipid droplet accumulation was qualitatively assessed using frozen-sectioned $(10 \mu \mathrm{m})$ placentae stained with Oil Red $\mathrm{O}$ and hematoxylin background. Images were captured using Nikon P90i microscope with NIS Elements software (Nikon Instruments Inc., Melville, NY, USA). Ten to twelve placentae from each group were used to quantify lipid content. Lipids and proteins were extracted simultaneously using a chloroform:methanol extraction from $100 \mathrm{mg}$ of homogenized full-placental sections taken adjacent to umbilical cord (through both maternal and fetal facing membranes). After centrifugation, the organic phase, containing lipids, was separated and dried down under nitrogen stream and reconstituted in $100 \mu \mathrm{l}$ $1 \%$ Triton-X. Because intracellular lipid droplets primarily contain neutral lipids, including diacylglycerol and TG (21), glycerol content was measured using the Triglyceride Colorimetric Assay described above.

\section{Placental mRNA Levels}

Using 10-12 placentae from each group, RNA was extracted from $30 \mathrm{mg}$ full-placental sections taken adjacent to the umbilical cord using the RNeasy Mini Kit (Qiagen, Germantown, MD). RNA integrity was assessed by electropherograms using 2100 BioAnalyzer (Agilent Technologies) and demonstrated RNA Integrity Numbers from 9.3 to 10.0. Placental RNA concentrations were between 242 and $561 \mathrm{ng} / \mu \mathrm{l}$ and were measured with NanoDrop $2000 \mathrm{UV}-\mathrm{Vis}$ Spectrophotometer (ThermoFisher Scientific). cDNA synthesis was performed using iScript cDNA Synthesis Kit (Bio-Rad, Hercules, CA) via the manufacturer's protocol. Primer pairs were manufactured at Life Technologies (Grand Island, NY) and quantitative realtime PCR (qPCR) was performed using Absolute Blue qPCR Mix (ThermoFisher Scientific) and analyzed with ABI 7500 System software (Applied Biosystems, Foster City, CA). Analyzed genes include glucose transporter 1 ( $S c l 2 a 1$ that encodes GLUT1), glucose transporter 3 ( $S c l 2 a 3$ that encodes GLUT3), hormone-sensitive lipase (Lipe that encodes HSL), lipoprotein lipase ( $L p l)$, FA translocase/ cluster of differentiation 36 (Cd36), FA-binding protein 3 (Fabp3), FA transport protein 1 (Scl27a1 that encodes FATP1), peroxisome proliferator-activated receptor gamma (Pparg), adipose differentiationrelated protein (Plin2 that encodes ADRP), and insulin-receptor substrate 1 (Irs 1$)$. Beta-2 microglobulin $(B 2 m)$ and tyrosine 3monooxygenase/tryptophan 5-monooxygenase activation protein zeta (Ywhaz) served as reference genes. Primer-probe information is shown in Supplementary Table S1 online.

\section{Placental Protein Expression}

Placental proteins were extracted as described above. After removal of the organic (lipid) phase, the protein was pelleted in methanol and dissolved in RIPA buffer overnight. After sonication of lysate, protein concentration was determined using a Pierce BCA protein assay kit (ThermoFisher Scientific). Proteins were resolved in $4-15 \%$ sodium dodecyl sulfate-polyacrylamide gel electrophoresis and transferred to polyvinylidene difluoride (PVDF) membranes using Trans-Blot Turbo RTA Midi PVDF Transfer Kit (Bio-Rad). Membranes were blocked in $5 \%$ bovine serum albumin/TBS-T followed by overnight incubation in primary antibodies against GLUT1, LPL, CD36, FABP3, and ADRP followed by $1-h$ incubation in horseradish peroxidase (HRP)-conjugated goat anti-rabbit IgG secondary antibody (SouthernBiotech, Birmingham, AL). Luminata Forte Western HRP substrate (EMD Millipore, Billerica, MA) and VisionWorks LS software (UVP, Upland, CA) were used for imaging and recording integrated optical densities. Readings were normalized to $\beta$-actin. Antibody information is shown in Supplementary Table S2.

\section{Hormone and Inflammatory Marker Analyses}

Maternal levels of circulating progesterone, estriol, and estradiol at E14 and E21 were measured via ELISA with the manufacturers' instructions (progesterone, MyBioSource, San Diego, CA, \#80558; estriol, Calbiotech, Spring Valley, CA, \#MBS021680; estradiol, 


\section{Articles | Lowwagie et al.}

Crystal Chem, Downers Grove, IL, \#ES180S-100). Levels were quantified using Cytation3 plate reader (BioTek Instruments, Winooski, VT).

Tumor necrosis factor alpha (TNF $\alpha$ ) and interleukin 6 (IL-6) levels were measured in serum from dams and offspring at P1 and E21 placental lysate using Milliplex Map Rat Adipocyte Magnetic Bead Panel (EMD Millipore, \#RADPCMAG-82K-02) with the manufacturer's instructions and quantified using Luminex 100/200 analyzer (Luminex, Madison, WI).

\section{Statistical Analyses}

Statistical analyses were performed using GraphPad Prism 6 (GraphPad Software, La Jolla, CA). Diet, diabetes, and interactionrelated effects were interrogated by two-way ANOVA. When interaction was significant, differences between controls and each exposed group were interrogated by one-way ANOVA and Dunnett's post hoc test. Changes in dam weight and TG levels over time were analyzed by linear regression analysis. Group data were averaged and expressed as means \pm SEM. A value of $P<0.05$ was considered significant.

\section{RESULTS}

\section{Model Characteristics}

Data are from 16 controls, 16 diabetes-exposed, 14 HF-dietexposed, and 14 combination-exposed litters. Of these, 11-13 dams/group (118-141 offspring/group) delivered naturally with outcomes measured on P1. Because dams consume placentae immediately upon delivery, an additional cohort with three to four dams/group (28-44 placentae/group) were delivered by C-section on E21 for placental collection. Although we have used this model for several years with consistent findings, some of the samples used for this study were from litters also used to previously report pulmonary and cardiac morbidity in newborn offspring $(13,14)$.

Consistent with previous studies using this model $(13,14)$, dams on a HF diet gained more weight than those fed control diet $(\mathrm{CD})$; the trend persisted over time $(P=0.001)$. Diabetic dams had higher whole-blood glucose levels after streptozotocin administration, whereas non-diabetic dams had normal levels regardless of diet; whole-blood ketones were higher in both diabetic and HF-fed dams (Table 1). Serum TG levels rose significantly with HF diet, especially after diabetes induction on E14 (Figure 1a) so that by P1 TG levels were twofold higher in diabetes-exposed, threefold higher in HFdiet-exposed, and 15-fold higher in combination-exposed dams (Table 1 and Figure 1b). Maternal NEFA levels also increased (Table 1 and Figure 1d,e).

Placentae and offspring characteristics are reported in Table 2. Placentae of diabetes- and HF-diet-exposed pregnancies weighed less. At E21 only diabetes-exposed offspring were smaller than controls, but at P1 both diabetes- and HFdiet-exposed pups were smaller. Fetal blood glucose at E21 (before severing the umbilical cord) was higher in diabetesexposed offspring and supra-additive in combination-exposed (interaction effect, $P=0.0001$ ). Notably, glucose levels in both dams and offspring rose significantly with surgery and anesthesia time so that fetal insulin levels more positively correlated with pup number than the treatment group;

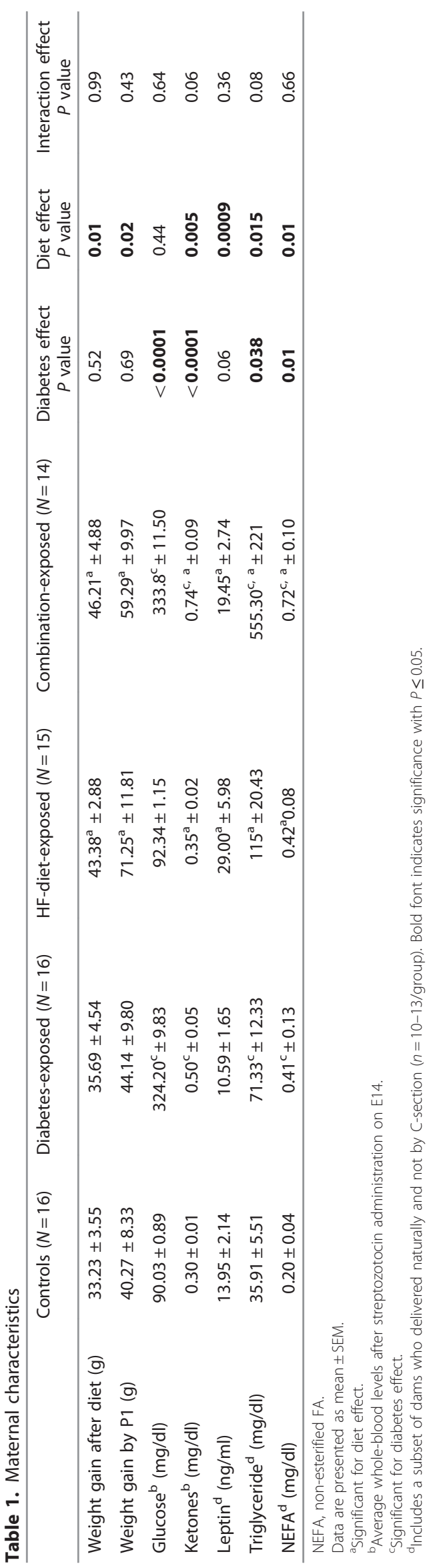



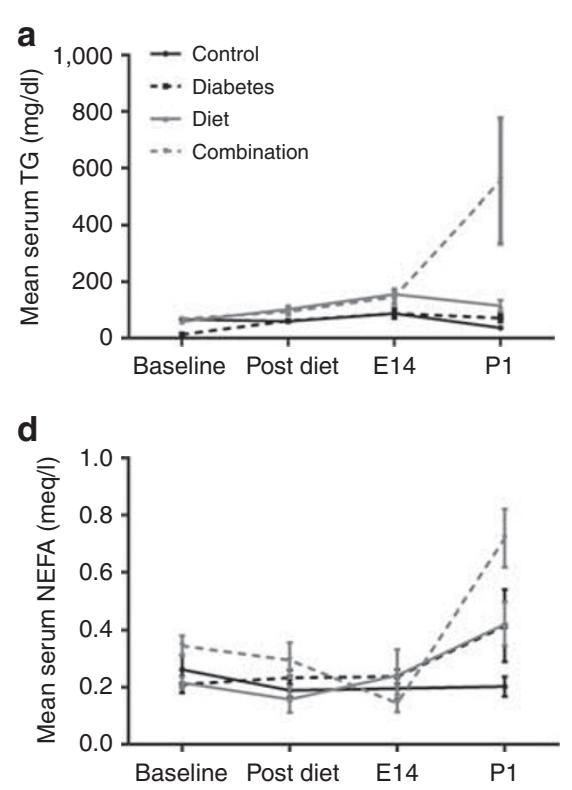
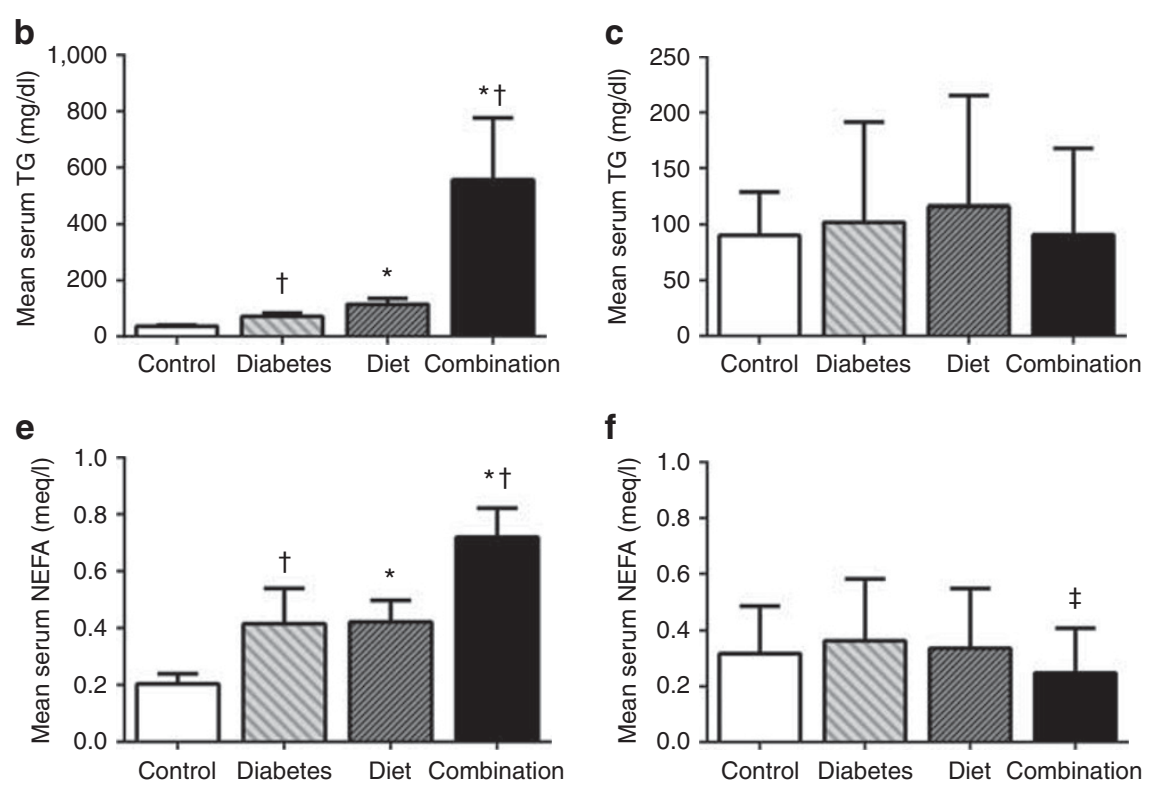

Figure 1. Maternal and newborn serum TG and NEFA levels. Graphs represent mean (SEM) serum TG (a-c) and NEFA (d-f) levels from controls, diabetes-exposed, high-fat diet-exposed, and combination-exposed rats. (a) Maternal TG levels at baseline, after 28 days of specified diet, embryonic day (E) 14, and postnatal day (P) 1 were higher in HF-diet-exposed dams after baseline and escalated in combination-exposed dams after diabetes induction at E14. (b) Maternal TG levels at P1 were significantly higher in diabetes-exposed and diet-exposed dams; combination-exposed dams had 15-fold higher TG than controls. (c) Offspring TG levels were not significantly different at P1. (d) NEFA levels in dams rise during the third trimester; this was more pronounced with diabetes and HF-diet exposure. (e) Maternal NEFA levels at P1 were significantly higher in diabetes- and HF-dietexposed dams. (f) Offspring NEFA levels were lower in combination-exposed pups. ${ }^{\dagger}$ Significant for diabetes and * diet effects by two-way ANOVA. ${ }^{\ddagger}$ Remains different than controls by one-way ANOVA with Dunnett post-test when a significant interaction is found; $P \leq 0.05$. HF, high fat; NEFA, nonesterified FA; TG, triacylglycerol.

therefore, they are not reported. Both diabetes-exposed and HF-diet-exposed P1 pups had higher circulating insulin levels.

\section{Circulating FAs}

Despite significant hyperlipidemia in diabetic and HF-fed dams, circulating lipid levels in offspring were not higher (Table 2 and Figure 1). Indeed, offspring TG levels were similar across groups and NEFA levels were potentially lower in combination-exposed pups $(P=0.01$ for interaction, $P=0.06$ by one-way ANOVA). Whole-blood FA composition (wt:wt\%) for P1 dams and corresponding offspring is reported in Table 3. In general, diabetic dams had higher fractions of saturated (palmitic), mono-unsaturated (palmitoleic and oleic acid), and 18-carbon precursor essential FAs (linoleic, LA, and $\alpha$-linolenic (ALA)) but lower fractions of stearic and ARA in circulation. Similar to their mothers, diabetes-exposed pups had a higher fraction of palmitoleic acid and lower fractions of stearic and ARA in circulation. HF-diet-exposed dams and pups had a higher composition of oleic acid likely reflecting dietary fat composition (Supplementary Table S3). However, they had lower fractions of circulating stearic and LA despite an increase in dietary provision. Although LCPUFA content was similar between diets, HF-diet-exposed pups had lower fractions of essential FA (LA, ALA, ARA, and EPA) regardless of maternal blood FA composition. True concentrations of whole-blood FA $(\mu \mathrm{g} / \mathrm{ml})$ were also measured in dams and corresponding P1 offspring (Supplementary Table S3).

\section{Placental Lipid Accumulation}

Lipid trapping in the placenta could explain the variation between maternal and fetal circulating lipids. Oil Red $\mathrm{O}$ staining demonstrates more lipid deposition in the decidua basalis (Figure 2a) and labyrinth zone (Figure 2b) of diabetes-exposed, HF-diet-exposed, and combinationexposed placentae. Both number and size of lipid droplets increased. Quantitative TG analysis confirmed lipid accumulation with $41 \%$ more $(P=0.01)$ in diabetes-exposed, $44 \%$ more $(P=0.004)$ in diet-exposed, and $69 \%$ more $(P=0.01)$ in combination-exposed placentae (Figure 3).

Relative differences in placental levels of key glucose and lipid transport and storage genes are shown in Figure 4. Scl2a3 (GLUT3), Cd36, Fabp3, and Scl27a1 (FATP1) mRNA levels were lower in HF-diet-exposed placentae. Pparg, Plin2 (ADRP), and Irs1 levels were lower in diabetes-exposed, HFdiet-exposed, and combination-exposed pregnancies. Most notably, combination-exposed placentae had 13-fold lower Irs1 than controls. Results were similar when validated using both $B 2 m$ and $Y w h a z$ as reference genes.

Placental protein expression was characterized using western blotting (Figure 5). Findings did not correlate with mRNA levels. GLUT1 protein expression was higher in diabetes- and HF-diet-exposed placentae. LPL expression was lower in HF-diet-exposed placentae. Despite lower mRNA levels, other protein levels were similar between groups. 


\section{Perinatal Mortality}

As previously reported $(13,14)$, a maternal HF diet, but not diabetes, was associated with a significantly higher perinatal mortality rate (Figure 6a). Indeed, HF-diet-exposed litters had a $41-43 \%$ perinatal mortality rate compared with only $9 \%$ in controls. Many of these losses occurred in utero (Figure 6b). Indeed, controls had $<3 \%$ stillbirth rate compared with $6 \%$ in diabetes-exposed, $30 \%$ in HF-dietexposed, and $21 \%$ in combination-exposed pregnancies. Findings emphasize the role of dyslipidemia in adverse pregnancy outcomes including pregnancy loss.

\section{Hormones of Pregnancy}

Maternal serum estriol, estradiol, and progesterone levels at E14 (before diabetes induction) and E21 are reported in Table 4. No significant dietary differences were found. However, at E21 diabetes-exposed dams had lower levels of estriol and progesterone. Although interesting, this cannot explain the higher stillbirth rate in HF-diet-exposed pregnancies.

\section{Markers of Inflammation}

Maternal, placental, and offspring TNF $\alpha$ and IL- 6 levels are reported in Table 5. No statistically significant difference was found, but P1 offspring had marked variability with a trend toward higher levels in both diabetes-exposed and HF-dietexposed offspring. Levels were only analyzed in live newborn offspring, which may confound data related to in utero mortality. Therefore, a conclusion about the role of lipids in inflammation related to perinatal mortality in this model cannot yet be reached.

\section{DISCUSSION}

This study uses a rat model to demonstrate that a HF diet exaggerates lipid-mediated consequences of a diabetic pregnancy. To our knowledge, we are the first to investigate individual and overlapping effects on placental lipid processing in relation to pregnancy maintenance and offspring health. We found evidence of placental lipid "trapping," and newborn offspring had lower fractions of circulating essential FAs that could have a negative impact on their short- and long-term health. Maternal HF diet, but not diabetes, was associated with higher perinatal mortality, especially stillbirths. Although lipid-mediated inflammation likely played a role (22), this could not be confirmed by this study alone. Together, findings highlight the negative consequences of dyslipidemia during pregnancy and demonstrate both protective and pathologic roles of the placenta.

Placental lipid accumulation (Figure 2) is an important finding in this study and confirms outcomes reported in other diabetic rodent models (23) and women (24). Our study adds that similar placental lipid accumulation occurs with a maternal HF diet and is even greater in combination with diabetes. Lipid accumulation may protect the developing fetus from lipotoxicity. Even when combination-exposed dams had circulating maternal TG levels 15-fold higher than controls, 
Table 3. Whole-blood FA composition (wt:wt\%) in dams and offspring

\begin{tabular}{|c|c|c|c|c|c|c|c|c|}
\hline \multirow[t]{2}{*}{ Fatty acid } & \multirow[t]{2}{*}{ Age } & \multicolumn{4}{|c|}{ Whole-blood fatty-acid composition (wt:wt\%) } & \multicolumn{3}{|c|}{ Significance } \\
\hline & & Controls & Diabetes-exposed & HF-diet-exposed & $\begin{array}{l}\text { Combination- } \\
\text { exposed }\end{array}$ & $\begin{array}{c}\text { Diabetes } \\
\text { effect }\end{array}$ & Diet effect & $\begin{array}{c}\text { Interaction } \\
\text { effect }\end{array}$ \\
\hline $16: 0$ & Dam & $21.99 \pm 0.29$ & $22.92 \pm 0.18 \uparrow$ & $19.02 \pm 0.85 \downarrow$ & $20.61 \pm 0.30 \downarrow$ & 0.02 & $<0.0001$ & 0.50 \\
\hline Palmitic & Offspring & $23.42 \pm 0.27$ & $24.14 \pm 0.47$ & $23.18 \pm 0.36$ & $23.76 \pm 0.49$ & 0.12 & 0.45 & 0.85 \\
\hline $16: 1$ & Dam & $0.27 \pm 0.02$ & $0.42 \pm 0.11 \uparrow$ & $0.27 \pm 0.05$ & $0.41 \pm 0.04 \uparrow$ & 0.04 & 0.93 & 0.98 \\
\hline Palmitoleic & Offspring & $0.75 \pm 0.07$ & $0.90 \pm 0.06 \uparrow$ & $0.85 \pm 0.04$ & $0.99 \pm 0.08 \uparrow$ & 0.04 & 0.15 & 0.91 \\
\hline Stearate & Offspring & $9.55 \pm 0.16$ & $8.91 \pm 0.22 \downarrow$ & $8.92 \pm 0.18 \downarrow$ & $8.56 \pm 0.26 \downarrow$ & 0.02 & 0.03 & 0.49 \\
\hline $18: 1$ n9 & Dam & $4.91 \pm 0.13$ & $6.42 \pm 1.15 \uparrow$ & $8.42 \pm 0.79 \uparrow$ & $12.10 \pm 1.30 \uparrow$ & 0.01 & $<0.0001$ & 0.27 \\
\hline Oleic & Offspring & $9.26 \pm 0.47$ & $11.08 \pm 0.46$ & $15.00 \pm 0.45 \uparrow$ & $15.25 \pm 0.81 \uparrow$ & 0.08 & $<0.0001$ & 0.19 \\
\hline $18: 2 n 6$ & Dam & $14.16 \pm 0.42$ & $16.12 \pm 1.47 \uparrow$ & $10.13 \pm 0.61 \downarrow$ & $13.13 \pm 0.46 \downarrow$ & 0.008 & 0.0004 & 0.55 \\
\hline $20: 4 n 6$ & Dam & $21.77 \pm 0.35$ & $19.49 \pm 1.08 \downarrow$ & $21.51 \pm 1.46$ & $16.61 \pm 1.09 \downarrow$ & 0.003 & 0.16 & 0.23 \\
\hline Arachidonic & Offspring & $21.52 \pm 0.40$ & $19.11 \pm 0.65 \downarrow$ & $17.87 \pm 0.28 \downarrow$ & $16.27 \pm 0.77 \downarrow$ & 0.001 & $<0.0001$ & 0.49 \\
\hline $20: 5 n 3$ & Dam & $0.43 \pm 0.01$ & $0.40 \pm 0.02$ & $0.35 \pm 0.03 \downarrow$ & $0.32 \pm 0.02 \downarrow$ & 0.23 & 0.003 & 0.94 \\
\hline Eicosapentaenoic & Offspring & $0.80 \pm 0.03$ & $0.78 \pm 0.02$ & $0.62 \pm 0.02 \downarrow$ & $0.64 \pm 0.02 \downarrow$ & 0.88 & $<0.0001$ & 0.52 \\
\hline $22: 6 n 3$ & Dam & $4.20 \pm 0.21$ & $4.20 \pm 0.16$ & $4.80 \pm 0.07 \uparrow$ & $4.43 \pm 0.24 \uparrow$ & 0.35 & 0.03 & 0.30 \\
\hline Docosahexaenoic & Offspring & $5.08 \pm 0.19$ & $4.41 \pm 0.19$ & $5.04 \pm 0.23$ & $5.08 \pm 0.24$ & 0.15 & 0.14 & 0.11 \\
\hline
\end{tabular}

FA, fatty acid; HF, high fat.

Whole blood FA composition is shown as mean wt:wt\% \pm SEM. Samples collected from dams ( $n=7 /$ group) and offspring ( $n=10-12$ from 6 to 7 litters/group) at postnatal day (P)1. Arrows demonstrate a significantly higher $(\uparrow)$ or lower $(\downarrow)$ FA composition in diabetes-exposed, HF-diet-exposed, and combination-exposed rats. Bold font indicates significance with $P \leq 0.05$ by two-way ANOVA for diabetes or diet effect.
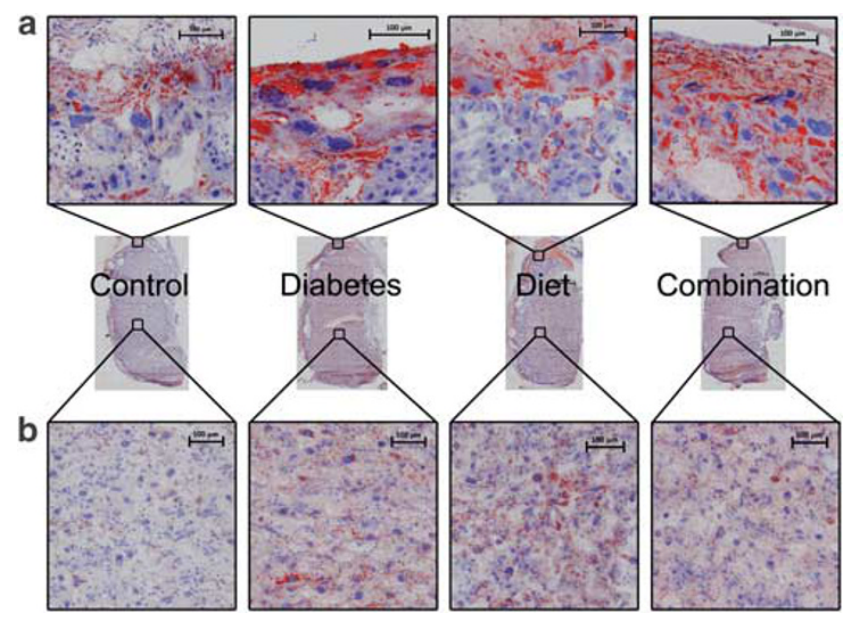

Figure 2. ORO staining of placental lipid droplets. Placentae from controls, diabetes-exposed, high-fat diet-exposed, and combinationexposed pregnancies were frozen-sectioned and stained with $\mathrm{H} \& \mathrm{E}$ background and ORO (red) to assess lipid deposition. (a) Decidua basalis (maternal-sided membranes). (b) Labyrinth zone (fetal-sided membranes). Lipid droplet size and number increase in diabetesexposed, HF-diet-exposed, and combination-exposed placentae. $\mathrm{Bar}=100 \mu \mathrm{m}$. H\&E, hematoxylin and eosin; HF, high fat; ORO, Oil Red O.

their offspring did not develop hyperlipidemia (Figure 1). However, in other tissues accumulation typically indicates pathology $(14,21)$, and placental lipid trapping may decrease

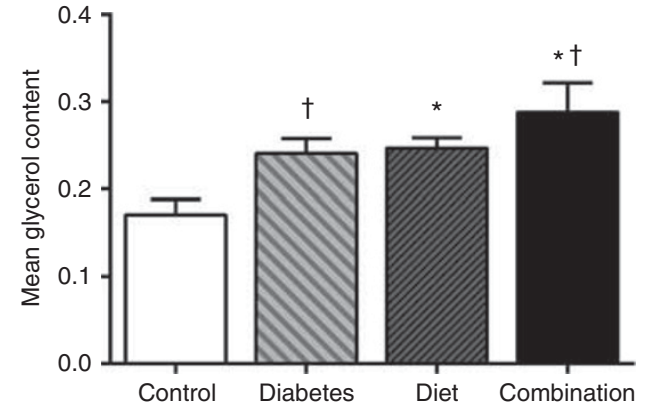

Figure 3. Placental glycerol content. Glycerol content, a marker of lipid droplet accumulation, is quantitatively higher in diabetes-exposed, highfat-diet-exposed, and combination-exposed placentae. Bar graphs represent mean (SEM) glycerol content in each group ( $n=8-14 /$ group). ${ }^{\dagger}$ Significant for diabetes and *diet effects by two-way ANOVA. $P \leq 0.05$.

provision of essential FA (including docosahexaenoic acid) to offspring (16-18). Despite our findings, we are not convinced that this is due solely to placental trapping. Rather, similar to their pups, diabetes-exposed dams in our study had lower ARA but higher LA composition, which suggests decreased in vivo conversion of precursor LA to ARA.

Expression of fuel uptake and storage genes has been studied as a marker of placental nutrient transport under diabetic conditions $(22,25-29)$. Our study supports previous reports (28) and extends findings to show that HF diet is also 


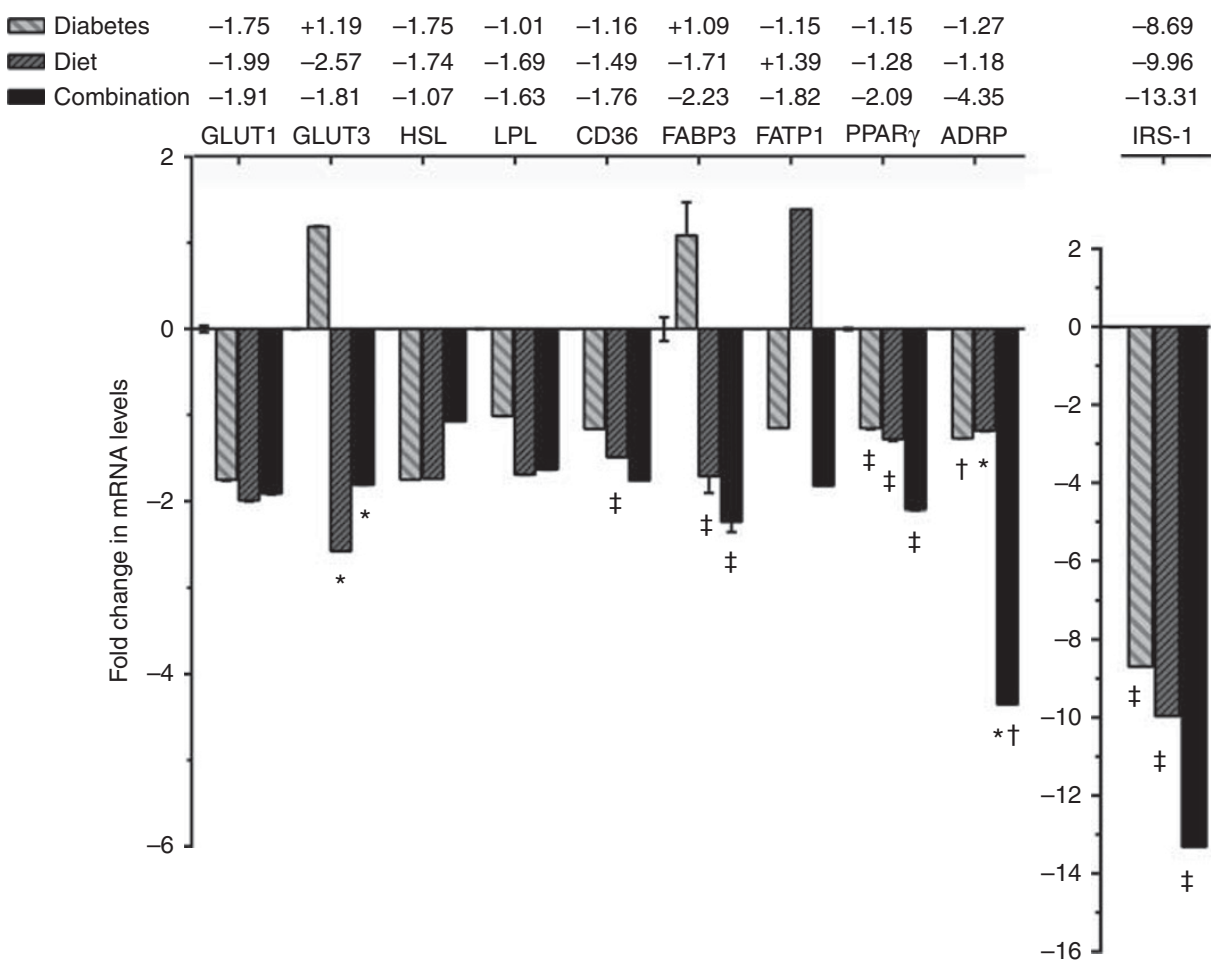

Figure 4. Placental mRNA levels of key fuel transport and storage genes determined using qPCR. Baseline (0) represents the mean mRNA levels of individual genes found in controls; B2m served as the reference gene. Bar graphs represent the mean fold change (SEM) in diabetes-exposed, highfat-diet-exposed, and combination-exposed placentae compared with controls ( $n=10-13 /$ group). ${ }^{\dagger}$ Significant for diabetes and *diet effects by twoway ANOVA. ${ }^{\ddagger}$ Remains different than controls by one-way ANOVA with Dunnett post-test when a significant interaction is found; $P \leq 0.05$.

associated with lower mRNA levels of key lipid transport and storage genes (Cd36, Fabp3, Pparg, Plin2 (ADRP), and Irs1). CD36, which transports hydrolyzed FAs into and out of cells, and FABP3, an intracellular FA transporter (30), are under transcriptional regulation by $\operatorname{PPAR} \gamma \quad(26,29)$, which is influenced by lipids and insulin. In humans, placental PPAR $\gamma$ protein expression is lower in women with gestational diabetes (31) but not obesity (30). In contrast, placentae from obese women have higher expression of CD36 than those from women of healthy weight (30). In our rats, both diabetes- and HF-diet-exposed placentae had lower mRNA levels of Pparg and downstream Cd36 and Fabp3. Leptin exposure increases CD36 protein expression in human placental culture (32), yet our in vivo model showed lower placental Cd36 mRNA levels despite higher maternal leptin levels. Differences could be species-specific, temporal (human placenta at 12 weeks' gestation (32) vs. term), or because HFfed dams gained weight without morbid obesity.

Although others have shown that mice on an extremely HF diet (60\% calories as fat) have higher Fabp3 expression (27), our findings are more consistent with human studies showing lower placental expression in obese women (30). Stable protein levels of CD36 and FABP3 among decreased mRNA levels with HF diet and combination exposure may indicate increased translation of gene products or decreased protein degradation. Additional study is needed to determine exact mechanisms of disparity.
LPL lies in maternal-facing placental membranes and hydrolyzes circulating TG to free FAs, allowing them to cross by passive or active transport (26). Lower $L p l$ mRNA levels could partially explain why HF-diet-exposed offspring have lower circulating lipid levels than dams, but expression does not necessarily predict LPL activity. Others have demonstrated increased LPL activity (without change in protein expression) in placentae from diabetic (33) and obese women (30).

Given that placental lipid droplets accumulate in diabetes and diet-exposed pregnancies despite lower mRNA levels of lipid transport genes, it is likely that this accumulation is due to decreased utilization rather than increased storage. Others support this plausible explanation $(34,35)$. The disparity in mRNA and protein levels of ADRP, a lipid droplet-associated protein, further supports this idea. Stable protein expression of ADRP despite decreased mRNA levels may represent less turn-over of lipid droplets in diabetes-exposed and HF-dietexposed rats; measurement of lipid droplet turnover would strengthen this explanation. IRS-1 plays vital roles in insulin signaling, insulin resistance, and inability to metabolize lipids (36). Thus, the marked decrease in Irs1 mRNA levels in combination-exposed placentae may incite metabolic dysfunction and lipid droplet accumulation. Indeed, offspring from this same model have cardiac lipid droplet accumulation and altered cellular bioenergetics, suggesting decreased utilization (14). As in our model, women with insulin-controlled 
a

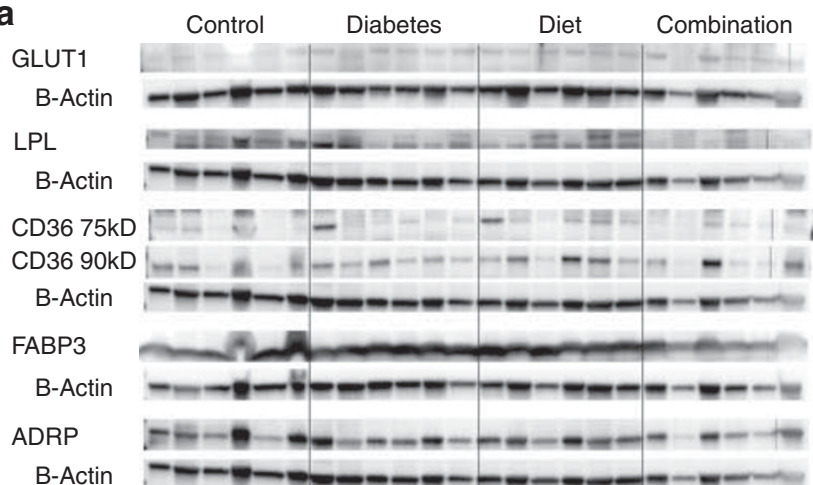

b

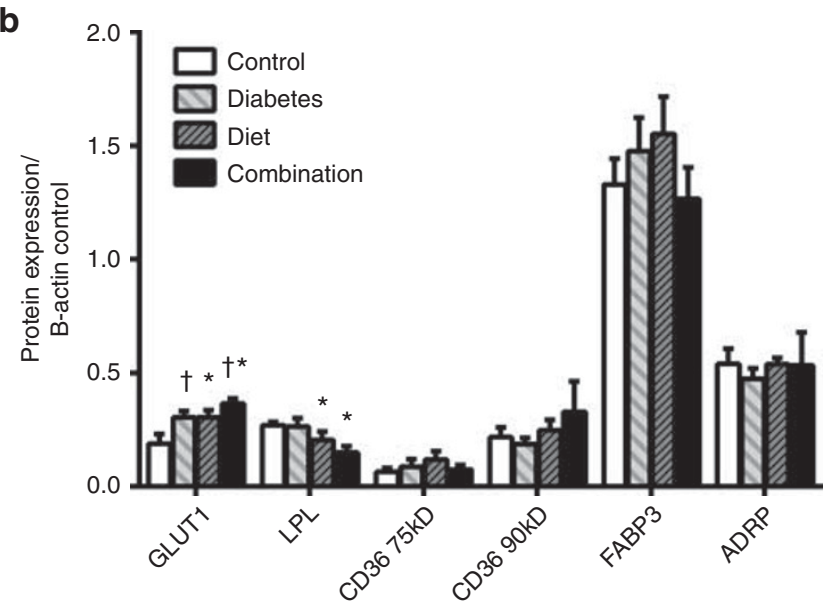

Figure 5. Placental expression of key fuel transport and storage proteins. Protein expression was determined using western blotting (a). Immunoblots of placental lysates for GLUT1, LPL, CD36 (two bands; both shown), FABP3, and ADRP are shown alongside corresponding blots for $\beta$-actin (loading control). Each lane represents equal protein contributions of multiple offspring ( $n=6 /$ group). (b) Mean (SEM) densitometry analysis of blots for each group. ${ }^{\dagger}$ Significant for diabetes and *diet effect by two-way ANOVA; $P \leq 0.05$. diabetes or obesity have lower levels of placental IRS-1 and evidence of placental insulin resistance (36).

Low estradiol and progesterone levels are associated with increased risk of spontaneous abortion (37); lower progesterone and estrogen concentrations are also seen in obese women (38). In our model, hormone levels were lower in diabetes-exposed, but not HF-diet-exposed dams that had the highest stillbirth rate; thus, impaired hormone production seems an unlikely cause of stillbirths in this particular model. In the rat placenta TNF $\alpha$ and IL- 6 serve as mediators of inflammation. Crew et al. demonstrated higher levels in placentae from rats fed a "cafeteria-style" diet (39). In our study, maternal, placental, and offspring levels of TNF $\alpha$ and IL- 6 trended higher in diabetes- and HF-exposed offspring. Only serum from live (not dead) newborn offspring was analyzed; therefore, we cannot exclude inflammation as a cause of in utero death. Given our findings, we suggest that future investigations clarify these points and expand focus on placental metabolism, reactive oxygen species production, and vascular flow.

\section{Strengths and Limitations}

This study allows direct examination of the effects of dietary fat intake and late-gestation diabetes on the maternalplacental-fetal triad independently and additively. Our rat model allowed strict dietary regulation and timing and control of diabetes while keeping physiologically relevant in vivo conditions. Our diet is both feasible and translational. Control and HF diets mirrored ideal and typical Westernized diets, with $18 \%$ and $40 \%$ caloric intake as fat with similar LCPUFA content. Although streptozocin-induced diabetes is neither Type I nor Type II, it allowed us to examine the effects of maternal hyperglycemia and fetal hyperinsulinemia in the third trimester. Our intentional timing eliminated confounding effects from early diabetes on placentation and organogenesis and corresponds to fetal pancreatic development,
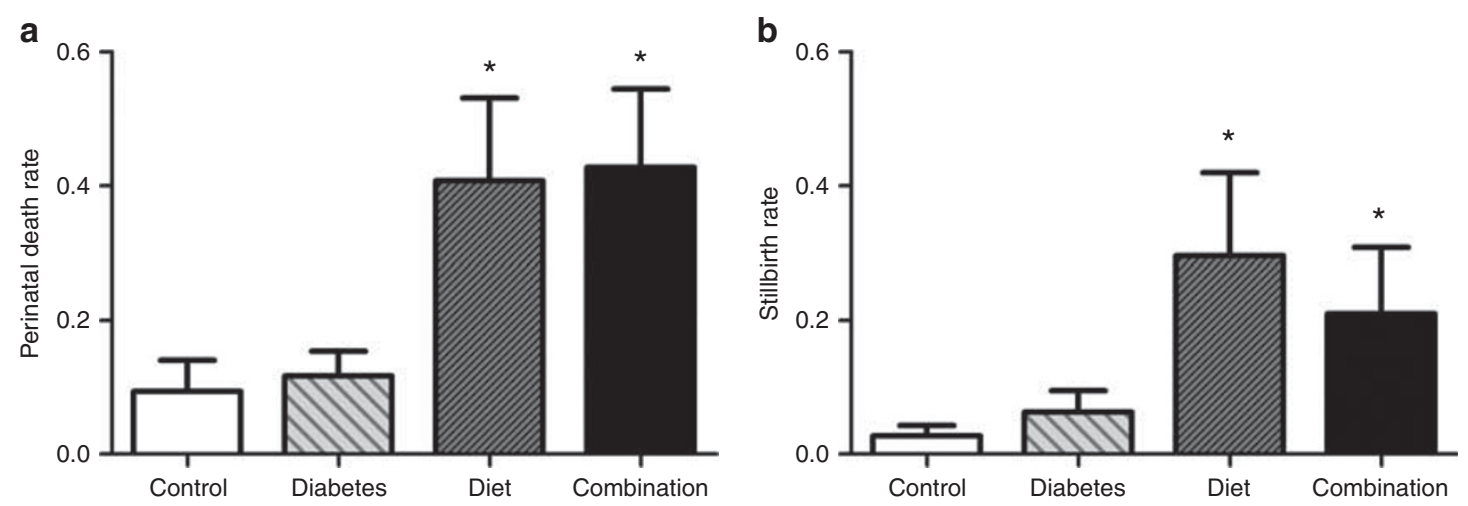

Figure 6. Perinatal loss. Litter size included all live and dead offspring that delivered on P1 with careful inspection of the uterine horns for obvious placentations/retained stillbirths. Bar graphs represent the mean (SEM) for controls, diabetes-exposed, high-fat diet-exposed, and combinationexposed pregnancies ( $n=10-13$ litters/group). (a) The perinatal mortality was calculated using the following equation: (total litter size - number of live offspring)/total litter size. (b) Stillbirth rate was calculated using the following equation: number of placentations/total litter size. High-fat diet, but not diabetes, was associated with significantly more perinatal deaths and stillbirths/litter. ${ }^{*}$ Significant for diet effect by two-way ANOVA; $P \leq 0.05$. 


\section{Articles | Louvagie et al.}

Table 4. Circulating hormones of pregnancy maintenance

\begin{tabular}{|c|c|c|c|c|c|c|c|c|}
\hline Hormone & $\begin{array}{l}\text { Time } \\
\text { point }\end{array}$ & Controls & $\begin{array}{l}\text { Diabetes- } \\
\text { exposed }\end{array}$ & $\begin{array}{l}\text { HF-diet- } \\
\text { exposed }\end{array}$ & $\begin{array}{c}\text { Combination- } \\
\text { exposed }\end{array}$ & $\begin{array}{c}\text { Diabetes effect } \\
P \text { value }\end{array}$ & $\begin{array}{c}\text { Diet effect } \\
P \text { value }\end{array}$ & $\begin{array}{l}\text { Interaction effect } \\
P \text { value }\end{array}$ \\
\hline \multirow[t]{2}{*}{ Estriol (pg/ml) } & E14 & $48.0 \pm 5.64$ & $105 \pm 20.5^{a}$ & $67.0 \pm 11.1$ & $50.2 \pm 14.8$ & NA & NA & 0.01 \\
\hline & E21 & $61.8 \pm 11.1$ & $37.0 \pm 6.8^{b}$ & $90.2 \pm 11.5$ & $42.7 \pm 9.2^{b}$ & 0.003 & 0.12 & 0.28 \\
\hline \multirow[t]{2}{*}{ Estradiol (pg/ml) } & E14 & $5.5 \pm 2.1$ & $6.5 \pm 4.6$ & $3.2 \pm 0.9$ & $2.2 \pm 0.4$ & 0.91 & 0.78 & 0.88 \\
\hline & E21 & $10.6 \pm 8.0$ & $16.8 \pm 8.3$ & $20.9 \pm 19.2$ & $18.7 \pm 9.8$ & 0.23 & 0.68 & 0.90 \\
\hline \multirow[t]{2}{*}{ Progesterone (ng/ml) } & E14 & $117 \pm 1.1$ & $116 \pm 2.8$ & $113 \pm 6.3$ & $118 \pm 1.1$ & 0.60 & 0.86 & 0.46 \\
\hline & E21 & $102 \pm 4.0$ & $96 \pm 4.8^{b}$ & $110 \pm 2.5$ & $101 \pm 2.1^{\mathrm{b}}$ & 0.049 & 0.06 & 0.67 \\
\hline
\end{tabular}

$\mathrm{HF}$, high fat; NA, when interaction present data is not significant by Dunnett post-test.

Maternal serum estriol, estradiol, and progesterone levels on embryonic day (E) 14 and 21 expressed as mean \pm SEM. Data are from four to five dams/group. Bold font indicates significance with $P \leq 0.05$.

aRemains different than controls by one-way ANOVA with Dunnett post-test when a significant interaction is found.

${ }^{\mathrm{b}}$ Significant for diabetes effect.

Table 5. Inflammatory markers of the maternal-placental-fetal triad

\begin{tabular}{|c|c|c|c|c|c|c|c|}
\hline & Controls & Diabetes-exposed & HF-diet-exposed & $\begin{array}{l}\text { Combination- } \\
\text { exposed }\end{array}$ & $\begin{array}{c}\text { Diabetes } \\
\text { effect } \\
P \text { value }\end{array}$ & $\begin{array}{l}\text { Diet } \\
\text { effect } P \\
\text { value }\end{array}$ & $\begin{array}{c}\text { Interaction } \\
\text { effect } \\
P \text { value }\end{array}$ \\
\hline \multicolumn{8}{|l|}{$T N F a(p g / m l)$} \\
\hline Maternal serum & $272 \pm 62(n=7)$ & $152 \pm 31(n=7)$ & $174 \pm 35(n=7)$ & $158 \pm 38(n=7)$ & 0.13 & 0.30 & 0.24 \\
\hline Placental lysate & $0.004 \pm 0.001 \quad(n=9)$ & $0.003 \pm 0.001(n=9)$ & $0.002 \pm 0.001 \quad(n=9)$ & $0.003 \pm 0.001(n=9)$ & 0.36 & 0.07 & 0.15 \\
\hline $\begin{array}{l}\text { Newborn } \\
\text { serum }\end{array}$ & $29.7 \pm 1.3(n=28)$ & $37.6 \pm 7.3(n=41)$ & $35.3 \pm 6.3(n=28)$ & $31.5 \pm 5.3(n=34)$ & 0.73 & 0.97 & 0.34 \\
\hline \multicolumn{8}{|l|}{ IL-6 (pg/ml) } \\
\hline Maternal serum & $715.4 \pm 137.8(n=7)$ & $359.4 \pm 114.8(n=7)$ & $416.8 \pm 104.5(n=7)$ & $334.67 \pm 111.5(n=6)$ & 0.078 & 0.19 & 0.26 \\
\hline Placental lysate & $0.1 \pm 0.001(n=9)$ & $0.009 \pm 0.001 \quad(n=9)$ & $0.007 \pm 0.001 \quad(n=9)$ & $0.009 \pm 0.001 \quad(n=9)$ & NA & $\mathrm{NA}$ & 0.027 \\
\hline $\begin{array}{l}\text { Offspring } \\
\text { serum }\end{array}$ & $2,125 \pm 163(n=28)$ & $2,867 \pm 319(n=41)$ & $2,152 \pm 366(n=28)$ & $2,315 \pm 254(n=34)$ & 0.13 & 0.38 & 0.33 \\
\hline
\end{tabular}

physiologic hyperlipidemia of pregnancy, and the typical timing of hyperglycemia in gestational diabetes. Measuring circulating FA composition and concentration was useful in further defining essential FA deficiency. However, we recognize that neither is a true measure of tissue FA accretion. Postpartum uterine horn examination to confirm placentations and unborn offspring was valuable in identifying the high stillbirth rate with HF-diet exposure.

Limitations include aforementioned differences in cause of diabetes and species-specific differences. Daily caloric intake and body fat composition were not measured. Although rats fed high caloric diets may limit food intake, this is often overcome with diets of impractically high-fat content $(\geq 60 \%)$. Our study used a more translatable fat content to study placental and fetal consequences resulting from high-fat diet rather than maternal obesity itself (Supplementary Table S4). Differences exist between rat and human placentae, but they share similar hemochorial structure with homologous fuel transport, storage, and signaling proteins including those in this study (40). Unlike humans, diabetes-exposed pups did not develop macrosomia. This may be related to differences in the length of gestation, litter size, placental size, or blood flow rates. These same factors could affect some of our measured outcomes including stillbirths, FA, and offspring cytokine levels. Measuring gene and protein expression is relative to expression of a reference, thus it is neither quantitative nor does it directly correlate to protein activity or overall nutrient transport that requires FA labeling (25). Moreover, protein expression results in this study should be taken in context of a low sample size, with further validations and investigation of post-translational modifications in any future work. Sexspecific differences were not interrogated and may also be important in offspring outcomes (22).

\section{CONCLUSION}

This study supports a growing body of evidence that maternal dyslipidemia has detrimental effects on the placenta and developing offspring. Specifically, a HF diet, especially alongside diabetes, was associated with higher perinatal mortality, placental lipid droplet accumulation, and altered 
FA profiles that may influence the health of developing fetus. Pathologic lipid droplet accumulation provides mechanistic clues that correlate with findings in humans and warrants additional investigation focused on placental metabolism, ROS production, adaption, repair, and vascular flow. Understanding the role of lipids in pregnancy sets the foundation for developing and implementing additional preventative measures to improve outcomes for the growing number of pregnancies affected by diabetes and obesity.

\section{SUPPLEMENTARY MATERIAL}

Supplementary material is linked to the online version of the paper at http://www.nature.com/pr

\section{ACKNOWLEDGMENTS}

We thank the Sanford Program for Undergraduate Research (SPUR) and Sanford Research, especially Andrew Cardillo, LARF staff, Claire Evans, Kelly Graber, Peter Vitiello, and Ben Forred for their significant support and help in many methods. M.L.B. is grateful to Jeffrey Segar and Andrew Norris, who serve as exceptional mentors and initially encouraged and supported the project ideas. M.L.B. also thanks Stacia Koppenhofer, Elizabeth Geisendorfer-Lindgren, and Jianrong Yao for technique training and validation that directly contributed to the project development. We are also grateful for input provided at the Midwest SPR and PAS during presentations by E.J.L.

\section{AUTHOR CONTRIBUTIONS}

M.L.B. and E.J.L. conceived the study, searched the literature, and designed the methods. E.J.L., T.D.L., A.L.W., and M.L.B. did the animal work. E.J.L. and T.D.L. with assistance from other authors validated and performed lipid extraction, glycerol and NEFA analyses, qPCR, western blotting, hormone ELISA, and Milliplex assays. T.D.L. and M.L.B. performed FA analyses. E.J.L., T.D.L., and M.L.B. did statistical analyses. E.J.L. and M.L.B. wrote the manuscript with review and editing by all authors. M.L.B. is the principal investigator responsible for the work as a whole and funded the project.

\section{STATEMENT OF FINANCIAL SUPPORT}

This study was financially supported by Sanford Research, National Institutes of Health (NIH), NICHD K08HD078504, SPUR/NSF REU DBI-1262744, and a Sanford School of Medicine-University of South Dakota Faculty Research Grant. Additional assistance was provided by the Sanford Research Molecular Genetics Core (NIH, NIGMS, and COBRE P20GM103620) and the Molecular Pathology Core (NIH, NIGMS, and COBRE P20GM103548).

Disclosure: The authors declare no conflict of interest.

\section{REFERENCES}

1. Sacks DA, Hadden DR, Maresh M, et al. Frequency of gestational diabetes mellitus at collaborating centers based on IADPSG consensus panelrecommended criteria: the Hyperglycemia and Adverse Pregnancy Outcome (HAPO) Study. Diabetes Care 2012;35:526-8.

2. Flegal KM, Carroll MD, Ogden CL, Curtin LR. Prevalence and trends in obesity among US adults, 1999-2008. JAMA 2010;303:235-41.

3. Freinkel N. Banting Lecture 1980. Of pregnancy and progeny. Diabetes 1980;29:1023-35.

4. Catalano PM, Hauguel-De Mouzon S. Is it time to revisit the Pedersen hypothesis in the face of the obesity epidemic? Am J Obstetr Gynecol 2011;204:479-87.

5. Nohr EA, Villamor E, Vaeth M, Olsen J, Cnattingius S. Mortality in infants of obese mothers: is risk modified by mode of delivery? Acta Obstet Gynecol Scand 2012;91:363-71.

6. Cundy T, Gamble G, Townend K, Henley PG, MacPherson P, Roberts AB.. Perinatal mortality in Type 2 diabetes mellitus. Diabetic Med 2000;17:33-9.
7. Langer O. Obesity or diabetes: which is more hazardous to the health of the offspring? J Matern Fetal Neonatal Med 2016;29:186-90.

8. Melamed N, Hod M. Perinatal mortality in pregestational diabetes. Int J Gynaecol Obstet 2009;104 (Suppl 1): S20-4.

9. Catalano PM, McIntyre HD, Cruickshank JK, et al. The hyperglycemia and adverse pregnancy outcome study: associations of GDM and obesity with pregnancy outcomes. Diabetes Care 2012;35:780-6.

10. Simeoni U, Barker DJ. Offspring of diabetic pregnancy: long-term outcomes. Semin Fetal Neonatal Med 2009;14:119-24.

11. Committee on Practice B-O. Practice Bulletin No. 137: gestational diabetes mellitus. Obstetr Gynecol 2013;122:406-16.

12. Cedergren MI. Maternal morbid obesity and the risk of adverse pregnancy outcome. Obstetr Gynecol 2004;103:219-4.

13. Baack ML, Forred BJ, Larsen TD, et al. Consequences of a maternal highfat diet and late gestation diabetes on the developing rat lung. PLoS ONE 2016;11:e0160818.

14. Mdaki KS, Larsen TD, Wachal AL, et al. Maternal high-fat diet impairs cardiac function in offspring of diabetic pregnancy through metabolic stress and mitochondrial dysfunction. Am J Physiol Heart Circ Physiol 2015;310:H681-92.

15. Harris WS, Baack ML. Beyond building better brains: bridging the docosahexaenoic acid (DHA) gap of prematurity. J Perinatol 2015;35:1-7.

16. Thomas BA, Ghebremeskel K, Lowy C, Offley-Shore B, Crawford MA. Plasma fatty acids of neonates born to mothers with and without gestational diabetes. Prostaglandins Leukot Essent Fatty Acids 2005;72:335-41.

17. Wijendran V, Bendel RB, Couch SC, Philipson EH, Cheruku S, LammiKeefe CJ. Fetal erythrocyte phospholipid polyunsaturated fatty acids are altered in pregnancy complicated with gestational diabetes mellitus. Lipids 2000;35:927-31.

18. Ghebremeskel K, Thomas B, Lowy C, Min Y, Crawford MA. Type 1 diabetes compromises plasma arachidonic and docosahexaenoic acids in newborn babies. Lipids 2004;39:335-42.

19. Roescher AM, Timmer A, Erwich JJ, Bos AF. Placental pathology, perinatal death, neonatal outcome, and neurological development: a systematic review. PLoS ONE 2014;9:e89419.

20. Wang ZYY, Xiang X, Zhu Y, Men J, He M. [Estimation of the normal range of blood glucose in rats]. Wei Sheng Yan Jiu 2010;39:133-7 42.

21. Thiam AR, Beller M. The why, when and how of lipid droplet diversity. J Cell Sci 2017;130:315-24.

22. Reynolds CM, Vickers MH, Harrison CJ, Segovia SA, Gray C. Maternal high fat and/or salt consumption induces sex-specific inflammatory and nutrient transport in the rat placenta. Physiol Rep 2015;3:e12399.

23. Martinez N, Capobianco E, White V, Pustovrh MC, Higa R, Jawerbaum A. Peroxisome proliferator-activated receptor alpha activation regulates lipid metabolism in the feto-placental unit from diabetic rats. Reproduction 2008;136:95-103.

24. Lindegaard ML, Nielsen LB. Maternal diabetes causes coordinated downregulation of genes involved with lipid metabolism in the murine fetal heart. Metabolism 2008;57:766-3.

25. Araujo JR, Correia-Branco A, Ramalho C, Keating E, Martel F. Gestational diabetes mellitus decreases placental uptake of long-chain polyunsaturated fatty acids: involvement of long-chain acyl-CoA synthetase. J Nutr Biochem 2013;24:1741-50.

26. Duttaroy AK. Transport of fatty acids across the human placenta: a review. Prog Lipid Res 2009;48:52-61.

27. Qiao L, Guo Z, Bosco C, et al. Maternal high-fat feeding increases placental lipoprotein lipase activity by reducing SIRT1 expression in mice. Diabetes 2015;64:3111-20.

28. Radaelli T, Lepercq J, Varastehpour A, Basu S, Catalano PM, Hauguel-De Mouzon S. Differential regulation of genes for fetoplacental lipid pathways in pregnancy with gestational and type 1 diabetes mellitus. Am J Obstetr Gynecol 2009;201:209 e1- e10.

29. Larque E, Demmelmair H, Klingler M, De Jonge S, Bondy B, Koletzko B. Expression pattern of fatty acid transport protein-1 (FATP-1), FATP-4 and heart-fatty acid binding protein (H-FABP) genes in human term placenta. Early Hum Dev 2006;82:697-701. 


\section{Articles | Louwagie et al.}

30. Dube E, Gravel A, Martin C, et al. Modulation of fatty acid transport and metabolism by maternal obesity in the human full-term placenta. Biol Reprod 2012;87:14 1-1.

31. Jawerbaum A, Capobianco E. Review: effects of PPAR activation in the placenta and the fetus: implications in maternal diabetes. Placenta 2011;32 (Suppl 2): S212-7.

32. Mousiolis AV, Kollia P, Skentou C, Messinis IE. Effects of leptin on the expression of fatty acid-binding proteins in human placental cell cultures. Mol Med Rep 2012;5:497-502.

33. Magnusson AL, Waterman IJ, Wennergren M, Jansson T, Powell TL. Triglyceride hydrolase activities and expression of fatty acid binding proteins in the human placenta in pregnancies complicated by intrauterine growth restriction and diabetes. J Clin Endocrinol Metab 2004;89:4607-14.

34. Borengasser SJ, Faske J, Kang P, Blackburn ML, Badger TM, Shankar K. In utero exposure to prepregnancy maternal obesity and postweaning high-fat diet impair regulators of mitochondrial dynamics in rat placenta and offspring. Physiol Genomics 2014;46:841-50.
35. Perazzolo S, Hirschmugl B, Wadsack C, Desoye G, Lewis RM, Sengers BG. The influence of placental metabolism on fatty acid transfer to the fetus. J Lipid Res 2016;58:443-54.

36. Colomiere M, Permezel M, Riley C, Desoye G, Lappas M. Defective insulin signaling in placenta from pregnancies complicated by gestational diabetes mellitus. Eur J Endocrinol 2009;160:567-78.

37. Lassance L, Haghiac M, Minium J, Catalano P, Hauguel-de Mouzon S. Obesity-induced down-regulation of the mitochondrial translocator protein (TSPO) impairs placental steroid production. J Clin Endocrinol Metab 2015;100:E11-8.

38. Burton GJ, Fowden AL, Thornburg KL. Placental origins of chronic disease. Physiol Rev 2016;96:1509-65.

39. Crew RC, Waddell BJ, Mark PJ. Maternal obesity induced by a 'cafeteria' diet in the rat does not increase inflammation in maternal, placental or fetal tissues in late gestation. Placenta 2016;39:33-40.

40. Chavatte-Palmer P, Tarrade A, Rousseau-Ralliard D. Diet before and during pregnancy and offspring health: the importance of animal models and what can be learned from them. Int J Environ Res Public Health 2016;13:586. 